\title{
Holonic Architecture of the Smart Grid
}

\author{
Ebisa Negeri ${ }^{1}$, Nico Baken ${ }^{1}$, Marjan Popov ${ }^{2}$ \\ ${ }^{1}$ Network Architectures and Services, Delft University of Technology, Delft, The Netherlands; ${ }^{2}$ Electrical Sustainable Energy, Delft \\ University of Technology, Delft, The Netherlands. \\ Email: e.o.negeri@tudelft.nl,n.h.g.baken@tudelft.nl, m.popov@tudelft.nl
}

Received January $14^{\text {th }}, 2013$; revised February $14^{\text {th }}, 2013$; accepted February $21^{\text {st }}, 2013$

Copyright @ 2013 Ebisa Negeri et al. This is an open access article distributed under the Creative Commons Attribution License, which permits unrestricted use, distribution, and reproduction in any medium, provided the original work is properly cited.

\begin{abstract}
With the growing concerns about sustainable energy, energy efficiency and energy security, the electrical power system is undergoing major changes. Distributed energy sources are becoming widely available at the lower parts of the grid. As a result, more and more end consumers are transforming from passive consumers to active "prosumers" that can autonomously generate, store, import and/or export power. As prosumers increasingly dominate the power system, the system demands capability that allows enormous number of stakeholders with heterogeneous types to exchange power on the grid. Unfortunately, the classical power system cannot efficiently handle this scenario since it was designed for centralized power distribution. Thus, restructuring the rather old power system is indispensable. In this paper, we apply the holonic approach to structure the smart grid as a system that is bottom-up organized from autonomous prosumers that are recursively clustered at various aggregation layers. Based on this, we present a control architecture of the smart grid using holonic concepts. Our control architecture is characterized by autonomy of the prosumers, distributed control, recursive self-similar control structures at different aggregation levels. Further, we present a service oriented architecture (SOA) framework that models the control functions that make up the holonic control architecture. Our proposed control architecture is tested using a simulation set-up.
\end{abstract}

Keywords: Smart Grid; Architecture; Holon

\section{Introduction}

The electric power system, a vital system supporting the economy of every nation, is undergoing tremendous transformations driven by the growing concerns for increasing the share of green power supply, as well as enhancing the reliability, security and efficiency of the system. As opposed to the classical power system where power is generated at large-scale power plants and transported in a unidirectional way to the distributed consumers, distributed energy sources (DES), such as solar panels and wind turbines, are increasingly penetrating into the lower parts of the power grid. With the availability of the DES, the end customers of the power system are transforming from passive consumers to active "prosumers" that can generate power on site and feed their surplus production back to the grid. According to the European parliament, all new buildings to be built after 2019 will have to produce their own energy on site ${ }^{1}$. Moreover, the foreseen massive presence of electric vehicles (EV) will have a huge

${ }^{1}$ European Parliament, “All New Buildings to be Zero Energy from 2019,” Committee on Industry, Research and Energy, Brussels, 2009. impact on the power system. The flexible appliances, energy storage systems, as well as the grid-to-vehicle (G2V) and the vehicle-to-grid (V2G) features of the EVs will offer the prosumers the flexibility to manage the shape of their loads. Given their generation capacity and their flexibility to shape their load profiles, the prosumers tend to autonomously manage their own resources and exchange/ trade power with their surrounding network. As prosumers dominate the system, the power system faces an era of "prosumerization" whereby enormous number and types of stakeholders can autonomously generate, store, import and/or export power. While in the classical power system power is transported in a unidirectional way topdown from the centralized plants to the distributed consumers, in the prosumer-dominated power system power will be primarily exchanged locally between the prosumers in a bidirectional way.

Apparently, restructuring the rather old power system is crucial in order to accommodate the dynamics. Accordingly, an advanced future power grid paradigm is envisaged, which is commonly referred to as the smart grid. The anticipated benefits of the smart grids are listed 
in the reports of the International Energy Agency (IEA) [1], the National Institute of Standards and Technology (NIST) [2] and the European Smart Grids Technology Platform [3]. Enhancing the reliability, security, efficiency, and resilience of the power system, accommodating all generation and storage options, enabling informed participation of customers, accommodating new products, services and markets are among the benefits. The challenge still remains on how to organize the smart grid in order to realize these benefits.

In this work, we focus on the future prosumer-dominated power system, and study how to organize the smart grids to deliver the expected benefits. We envisage to organize smart grids bottom-up from prosumers. While prosumers autonomously manage their own resources, they can join other prosumers whose profile "complement" their own to exchange power with them. Further, a network of such prosumers might constitute a larger prosumer cluster to gain the power of the collective and bargain with the rest of the grid as a unit. Yet, several clusters of prosumers can form a network at a next aggregation level to form a bigger prosumer cluster and benefit the power of the bigger collective. In this context, it seems that the future power system builds bottom-up through recursive clustering of prosumers at various aggregation levels forming networks of networks. We also propose a suitable control architecture for such a bottom-up organized smart grid. To this end, we embark on concepts from holons and holarchy, which will be described in Section 3.

The contributions of this paper are summarized as follows:

- We employ the holonic concepts to organize the smart grids enabling it to foster seamless accommodation of the foreseen dominance of prosumers.

- We propose a holonic control architecture for our envisaged smart grids.

- We present an SOA framework to support our proposed control architecture and validate it with a simulation set up.

The remainder of this paper is organized as follows. In Sections 2 and 3, we present the related work and the concepts used in our work, respectively. The envisaged smart grid holarchy and the holonic control architecture are described in Sections 4 and 5, respectively. After presenting our simulation scenario and results in Section 5 , we provide discussions and concluding remarks in Section 6.

\section{Related Work}

Recently, various new concepts and developments have been proposed to manage the new trends in the power system. The concept of virtual power plants (VPP) was introduced as a cluster of distributed power sources which are collectively managed [4]. The Microgrid concept [5] was also suggested as a low voltage (LV) distribution system comprising of distributed sources, controlled loads and storage systems that are coordinated to achieve a controllable operation either as an island or connected to the medium voltage (MV) grid.

Provoost et al. [6] introduced the autonomous network (AN), which is a part of the system but its behavior is more or less independent from the rest. AN differs from the microgrid because AN is larger in size and complexity, as well as its aim is primarily optimizing its normal operation. Overbeeke and Roberts [7] proposed the $a_{c}$ tive networks concept, where the network is subdivided into cells that are self managing, but not necessarily self supplying. This concept involves interconnection between the cells that provides more than one power flow paths, and allows rerouting to avoid congestion and to isolate faults. Further, system services are traded along the connections between the cells.

The smart grid concept represents the overall picture of the future power network that is supported by intelligent distributed devices and communication technologies. Tsoukalas and Gao [8] modeled the smart grid as an energy internet comprising of local area grids (LAGs), that are demand based autonomous entities consisting of a convenient mixture of different customers. Huang et al. [9] presented the Future Renewable Electric Energy Delivery and Management (FREEDM) as a power distribution system that interfaces with residential customers and industry customers having distributed renewable energy sources and distributed storage devices. The key technology features of the system are the plug-and-play interface, energy router, and pen-standard based operating system. The proposed system relies on a flat and distributed management architecture.

A market based control concept, named PowerMatcher, is suggested by Kok et al. [10] for supply and demand matching in electricity networks with large penetration of distributed sources. In the PowerMatcher, each device uses its agent to buy or sell power in the electronic market that is implemented in a distributed manner using a tree structure. On the other hand, service oriented architecture (SOA) is gaining attention in power system control. Vaccaro et al. [11] have proposed a web-servicebased framework for integrated microgrid (MG) control, modeling, and monitoring. At the core of their framework is the MG engine which executes the MG management in a geographically distributed scenario.

Although their scale, complexity and intelligence varies, all the above concepts and technologies have their own contributions in managing the distributed sources. One concept could be more applicable in some settings than the other, and vice versa. Moreover, some of the concepts could be combined since they complement each 
other. The smart grid could be an umbrella to appropriately combine the concepts, thereby achieve a heterogeneous and intelligent power system that delivers its expectations. For instance, the FRIENDS, VPP, microgrid, AN, and active networks could form sub-networks of the smart grid, while FREEDM provides the power delivery infrastructure supporting the sub-networks and PowerMatcher delivers the transaction services.

In this work, we study how the smart grids can be organized to accommodate the foreseeable dominance of prosumers. We endeavor to propose an architecture of the smart grid that tends to converge the various proposed concepts. We model the heterogeneous types of components of the smart grid as prosumers. Based on this, we propose to organize the smart grid bottom-up from prosumers, by recursively clustering them at various aggregation layers. Moreover, we also propose a control architecture that fits our proposed organization of smart grids. Our prosumer model and control architecture are built based on the concept of holons and holarchy that is quite suitable to capture the properties of the future smart grids.

\section{Related Concepts}

\subsection{Prosumer}

In this work, prosumer is a general term that refers to a system that autonomously manages its resources and is capable of bidirectional exchange of power with its surrounding network. A prosumer could be as simple as a household that autonomously manages its resources (such as appliances, distributed sources, storage systems, electric vehicles, etc.). Other systems such as a virtual power plant, a microgrid, an energy community, an autonomous network, a power plant, etc. can also be regarded as prosumers. A prosumer can comprise sub-parts that are themselves prosumers, while at the same time can become a part of a larger prosumer. With the aid of the intelligent information and communication technology (ICT) capabilities of the smart grid, a prosumer can orchestrate its resources as well as coordinate itself with its surunding network to achieve desirable internal and system wide goals.

\subsection{Holon and Holarchy}

The word holon was coined by Koestler [12], and it refers to a logical entity that is both a whole and a part. For example, a cell in your body is a holon because it is a disnct living entity that has a distinct cell wall defining its interface with the rest of the world. However, a cell is composed of smaller holons such as nucleus and chromosome that are also separate entities. Yet, a group of cell holons together form a higher aggregation layer tissue holon, a group of tissue holons form an organ holon, and so on.

A holon is basically autonomous, cooperative and recursive. Holons exhibit self-similar recursive structure and are organized in various aggregation layers to form a holarchy as shown in Figure 1. Holarchies are recursive in the sense that a holon in a holarchy can in itself be an entire holarchy of other holons. In a holarchy, each holon can function autonomously, thereby enabling formation of a distributed system. While functioning autonomously, a holon can cooperate with other holons in the holarchy to achieve mutual goals. In a dynamic environment, holons can also be dynamically reorganized, thereby making the holarchy more robust to changes adapting itself to its environment. These properties of the holonic concept make it quite appealing to organize very complex systems. The holonic approach has been proposed to organize various systems, some of them are organizational modeling, manufacturing, sensor networks, traffic light management, etc. ([13-17]).

\subsection{Service Oriented Architecture (SOA)}

SOA is an architecture that models an application as a set of loosely coupled, flexible, reusable and adaptable functional units called services, whereby the services are interrelated through well-defined interfaces and contracts. The main advantages of SOA include platform-independence of the interfaces that aids interoperability, the flexibility and reusability of the services, and its easy adaptation to evolutionary changes in the application. While SOA can be implemented using different technologies, the web services are the most popular one because of wide acceptance of its standards. Web service technology exposes web services, which are sets of operations on the web; thereby allowing high degree of integration among them. These features of SOA and web services technologies are attractive to model the control functions of the holonic control, as will be discussed later.

\section{The Holonic Smart Grid}

While the smart grid has diverse types of components, such as households, microgrids, energy communities, etc.,

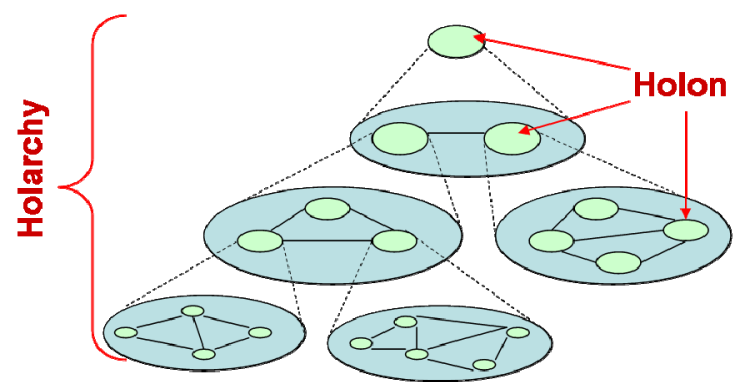

Figure 1. A holarchy-organization of holons. 
each of the components can be seen as a prosumer. The prosumers tend to autonomously manage their resources. Moreover, they tend to aggregate together and form a bigger cluster to facilitate local power exchange as well as to gain a larger power of the collective to trade power with their surrounding. For instance, a group of prosumer households can cluster together to form a neighborhood energy community, which is a larger prosumer. Likewise, a group of neighborhood energy communities can form a district energy community (yet a larger prosumer) at the next aggregation level. This clustering could further be recursively repeated at various aggregation levels. Moreover, the prosumers can dynamically reorganize to efficiently adapt to the change in their environment. Apparently, the autonomy, aggregation into layers, recursive property, and dynamic adaptation of the prosumer based smart grids closely matches the properties of a holonic system. Accordingly, we model each prosumer as a holon and the entire smart grid as a holarchy. The smart grid holarchy has the following major features that contribute to the overall efficiency of the system.

\subsection{Autonomy/Self Management}

As prosumers own production capacity and acquire the flexibility to manage their own load profiles, they tend to autonomously manage their resources to optimize their interest. This is in line with the growing interest towards active participation of customers. A prosumer holon might also choose to be part of a bigger prosumer holon and exchange power with its surrounding, or operate as a self-supplying islanded unit. The autonomy of the prosumer holons aids a distributed control capability of the system that would be otherwise very difficult to control centrally. Autonomy might also increase the consciousness of the prosumer holon, thereby making it more cautious about its consumption. Autonomy of the prosumers might make coordination not easy to achieve, but this can be achieved using machine learning [18], game theory [19] and mechanism design [20] based approaches.

\subsection{Recursive Aggregation}

As mentioned earlier, aggregation is important for prosumers to facilitate local power exchange between themselves, and to gain a bigger bargaining power with the rest of the grid. Accordingly, the prosumer holons at a lower aggregation level may be self-organized to constitute a bigger prosumer holon. For the same reasons, the newly formed prosumer holon can still be connected to its peers in a network in the next higher aggregation layer to form yet a bigger prosumer holon. Further bottom-up grouping of such holons in networks-of-networks continues recursively in higher aggregation levels and eventually constitute the overall holarchy of the smart grid
(Figure 2). Simon [21] has shown that this recursive clustering at different aggregation layers is inherent behavior of all complex systems. We refer to the smaller holons that form a bigger holon as the sub-holons of the bigger holon, whereas, the bigger holon on the next aggregation layer that contains a holon is referred to as its super-holon.

The bottom-up organization at different aggregation layers provides efficient structure that encapsulates and simplifies the coordination of the system, making it scalable. Moreover, the aggregation of prosumer holons into super-holons might increase the reliability of the collective as the profiles of the individual holons might complement each other. Further, the local power exchanges between the prosumer holons reduce both the transportation losses and the costly investment costs to upgrade the transmission capacity to meet the ever increasing demands.

\subsection{Dynamic Reconfiguration}

Over an interval of time, a prosumer holon may be in the state of either self sufficiency, surplus production, or excess demands. Thus, the prosumer holons can leave one super-holon to join another one over a period of time to look for a set of holons that complement their profiles, thereby optimizing their resource utilization. Erol-kantarci et al. [22] have shown that a set of microgrids could be dynamically reorganized during a day into clusters according to their profiles to optimize the utilization of their renewable resources. Moreover, prosumer holons can join a super-holon for some time intervals during a day and operate in islanded mode at other intervals, for example in response to potential faults. Such flexibilities lead to a dynamic reconfiguration of the smart grid holarchy over a period of time. The dynamic reconfiguration contributes to the self-healing, dynamic recovery, and increase efficiency of the prosumer holon.

\subsection{Intelligence and Communication}

A prosumer holon needs to coordinate its components, as well as coordinate itself with its surrounding to optimize utilization of resources and to ensure system stability.

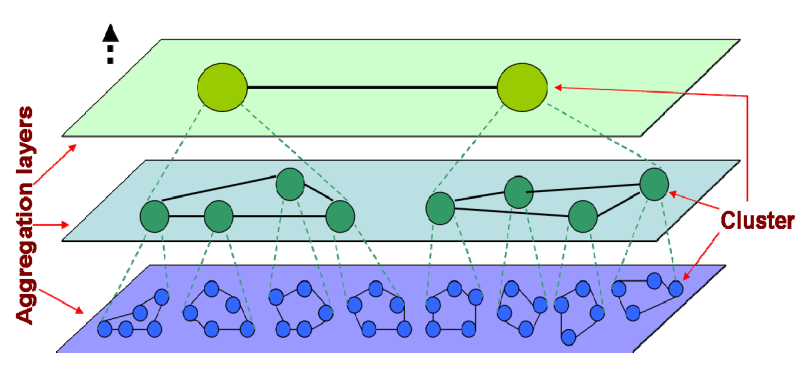

Figure 2. Bottom-up organization of the prosumers to form the smart grid holarchy. 
This coordination involves large information flows and requires intelligent information processing units that constitute the "nerve system" of the "organic" power system. Accordingly, each prosumer holon requires communication capabilities and intelligence to make decisions based on the gathered information. Indeed, an appropriate communication infrastructure needs to be in place. The IEC61850 standard [23] is developed for communication in substations. A review of the types of intelligence required as well as the candidate communication technologies for smart grids is presented in [24].

\subsection{Security and Privacy}

Cyber security is regarded as one of the biggest challenges in smart grids [2,3]. The enormous exchanges of information and control messages raise security concerns for the prosumers. Thus, the information and control message exchanges need to be protected against unauthorized access and guarded from malicious hackers. Therefore, the system requires a secure communication protocol. Moreover, privacy is becoming a major concern as the data exchanged might reveal personal information such as habits, activities, and preferences of the individual. For instance, the study in [25] has revealed that personal information can be estimated with high accuracy based on the meter reading of a household, even with relatively unsophisticated hardware and algorithms. Several solutions have been proposed to overcome security and privacy issues in smart grids, and their review is presented in [24]. The IEC61850 standard also supports secure communication in substations.

\section{The Holonic Control Architecture}

Given the nature of the envisioned smart grid, its control architecture needs to exhibit the following properties: 1) sufficient autonomy of each prosumer holon to manage its own resources; 2) recursive structure where a prosumer is composed of smaller prosumers at the lower aggregation layer, and at the same time be part of a larger prosumer holon at a higher aggregation layer; 3) layered structure to facilitate coordination between different aggregation layers; 4) dynamic reconfiguration to runtime topology change. The holonic architecture, as explained before, nicely fits the envisioned system because it possesses all of the properties required by the control architecture.

Our holonic control architecture maintains the proposed structure in the envisioned power system. In the control domain, a control-holon represents the control system of a prosumer holon. Thus, holonic control units will be recursively organized in a bottom-up structure to form a complete control holarchy of the smart grid. When the prosumer holon has prosumer sub-holons, its controlholon is also composed of the corresponding control-subholons (i.e. control systems of sub-holons).

In addition to controlling the internal dynamics of its prosumer holon, a control-holon needs to interact with the control-holons of its peer prosumer holons that are in the same super-holon as well as with that of its super-holon. Moreover, a control-holon needs to interact with the network operator operating at its aggregation level, regarding the power quality, infrastructure constraints, etc. Thus, the control functions of the control-holons are basically the same independent of their aggregation levels. This reveals the self-similarity (recursive property) of the control holarchy. Figure 3 shows the functional architecture of a single control-holon. Corresponding to each of the control functions in the control architecture shown in Figure 3, we define a corresponding web service in the SOA model together with its possible operations. The control functions and their corresponding web services

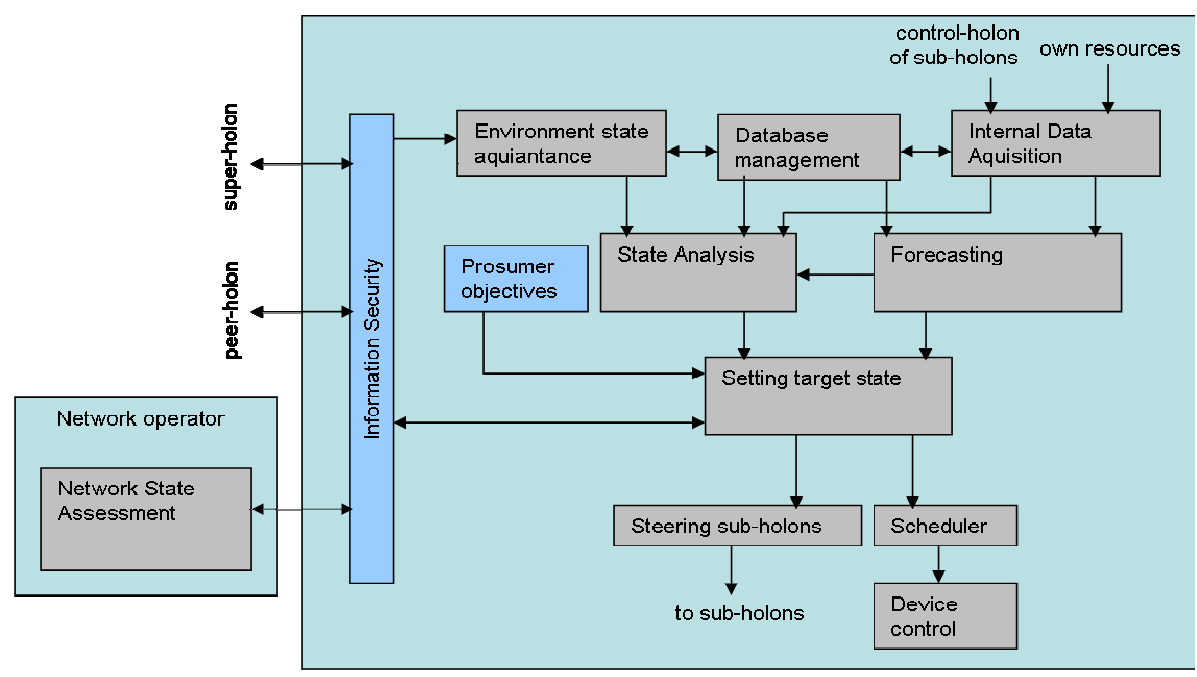

Figure 3. Architecture of a control holon. 
are described subsequently.

\subsection{Environmental State Acquaintance}

A prosumer holon needs to be well acquainted with its environment in which it is operating to make optimal decisions. Acquiring information about the load profiles of its peer prosumers, market prices, incentives, etc. is helpful to plan optimal transactions with its environment. When a super-holon sets a target state (of, for instance, the aggregate load profile), it might generate control signals, incentives, etc. for its sub-holons, based on their mutual agreements, to achieve the desired goal. Therefore, this control function is responsible for acquiring such information and presents it to the relevant control functions in a useful shape. The web service corresponding to this control function is EnvironmentSA-srv. To provide its functionality, the web service involves several operations. Some of the operations that compose the EnvironmentSA-srv web service are GetLoadProfile-opr, to acquire the load profile in its environment, and GetMarketState-opr, to acquire the market price in its environment.

\subsection{Internal State Acquisition}

A prosumer holon needs to have up-to-date information about its own constituents in order to make optimal decisions. This function handles acquiring internal data about the parameters of the resources of the prosumer (such as distributed generations, storage systems, etc.), their current available capacities, the state (such as, the load profiles, surplus productions, etc.) of the prosumer sub-holons, etc. Corresponding to this control function, we define the InternalDA-srv web service. Below are some of the operations constituting the web service:

- GetResourcePar-opr: to get the parameters of a resource, such as the storage capacity of a battery.

- GetResourceState-opr: to acquire the current state of a resource, e.g. the current charge level of a battery.

- GetSubProfile-opr: to get the load profile of a prosumer sub-holon.

\subsection{Database Management}

This function is responsible for storing, retrieving and updating the relevant internal and external data. The web service that provides this function is DatabaseMgt-srv. Accordingly, the following operations can be identified as some of its components: RdData-opr, RtData-opr, UpData-opr, and DIDalata-opr, that are used to read, write, update, and delete data, respectively.

\subsection{Forecasting}

This control function takes care of forecasting both the internal profiles such as generation profile of its local power sources, its local power demands, the load profile of its sub-holons (if any), as well as the load profile of its environment. The equivalent web service is Forecast-srv, which could compose the operations ForecastProduction-opr, ForecastDemand-opr and ForecastEnv-opr that are responsible for forecasting the local production, local demand, and the load profile of its environment, respectively.

\subsection{State Analysis}

Based on the inputs from the environment state acquaintance, the internal data acquisition, and the forecasting control functions, this control function analyzes the resulting state of the system. This allows the control holon to assess the possible load profiles, the flexibility in the load profiles, the synergetic potential of the various components of the resources and the load profiles, etc. We define StateAnalysis-srv as the web service that takes care of this control function. The following are some of the operations that could compose this service:

- AssFlexibility-opr: to assess the flexibility of the load profiles.

- AssSynergy-opr: to assess the synergetic potential of the resources.

- CompState-opr: to compute possible states of the aggregate load profiles of the holon.

\subsection{Setting Target State}

After acquiring the state estimation information, this function sets the target state for the prosumer. The target state specifies the target load profiles, amount of power to be imported/exported, whether to operate connected or as an island (where applicable), etc. To set the target set, the control function uses as input, among others, the objectives of the prosumer, which could be cost minimization, increasing comfort, increase composition of green energy, etc. The web service that handles this function is TargetState-srv, and some of the operations that compose it are SetTargetProduction-opr and SetTargetDemand-opr that take care of setting the target production and demand profiles, respectively, of the prosumer holon.

\subsection{Steering Sub-Holons}

Based on the target state proposed, this control function generates relevant signals for the sub-holons that would help to achieve the goal. The signals generated could be incentives or control signals depending on the type of the terms and conditions in the contract of the prosumer with its sub-holons. Corresponding to this control function, we define a web service called SteerSubHolon-srv. The operations that constitute the service could be GenControlopr and GenIncentive-opr to generate downstream control 
signals and incentive signals, respectively, for its sub-holons.

\subsection{Scheduler}

The scheduler determines the optimal load profiles of the schedulable resources of the prosumer to achieve the target state. Its equivalent web service is Scheduler-srv. An operation that could make up the web service is GenSchedule-opr, which is used to generate the optimal schedule of the resources.

\subsection{Device Control}

The device control function translates the schedule of the resources into the actual control signals of the devices that are scheduled. The DeviceControl-srv web service takes care of this, while the operation SetDeviceControlopr could be employed to set the device controls accordingly.

\subsection{Information Security}

The control function maintains the security of the information and control message exchanges of the controlholon with its environment. Its corresponding web service is Security-srv, which could have the following operations.

- EncryptInfo-opr: to encrypt the information to be sent.

- DecryptInfo-opr: to decrypt the received information.

- CheckAuthorization-opr: to check the authorization of the sender of the information.

\subsection{Network State Assessment}

This control function takes care of assessing the state of the power network over which the prosumer holons are interconnected. This includes analyzing the voltage levels at various nodes in the network, the loading of the infrastructures, etc. It also deals with contingency analysis, alarms about faults and potential risks in the environment. This functionality is provided by the network operator through the web service NtkStateAssessment-srv, which could be composed of operations such as, AssessNetk-opr, to assess the state of the network, and SdAlarms-opr, to send alarms to the corresponding holons.

\section{Simulation Setup}

We considered a system where our proposed control architecture can be applied. Our experimental setting consists of prosumer holons that are aggregated into three layers. At the lowest level are a set of prosumer households $(\mathrm{PH})$ that form a neighborhood energy community at the next aggregation level. The neighborhood energy community (NEC) is in turn part of a district energy community (DEC) at the next higher aggregation layer. Al- though their aggregation levels differ, the PHs, the NEC, and the DEC are all autonomous prosumer holons. The control-holon of the $\mathrm{PH}$ autonomously manages its resources to achieve a desired load profile of the $\mathrm{PH}$. The control-holon of the NEC coordinates the control-holons of its PHs, by providing incentives, to achieve a desirable aggregate load profile that maximizes the benefit of the NEC in response to the incentive received from the control-holon of DEC.

The power flow in the power grid is managed by a network operator (NO). The NO interacts with the relevant control holons to make sure that the power flows in the network do not violate the infrastructure constraints. Further, it generates appropriate control signals for the relevant control-holon whenever a fault occurs in the network.

The sketch of the interconnection between the described holons is depicted in Figure 4. The figure shows both the physical grid connection (solid lines) and the corresponding network of the control-holons (doted lines). The control-holons $(\mathrm{CH})$ in the figure could be implemented in intelligent devices that are capable of both making intelligent decisions and communicating across a communication infrastructure. For example, the $\mathrm{CH}$ of a prosumer household can be implemented in the home energy management system [26]. In our experimental setting, the control-holons are hosted by computers that are interconnected by IP network.

\section{A Case Example}

We present a case example that is implemented in the setting described above. Load management aiming at peak load reduction in a NEC that is composed of PHs is considered. Each of the PHs owns a solar panel, a micro-CHP unit, an electric vehicle and other electricity consuming appliances. The DEC wants to minimize its aggregate peak load and uses a control signal to steer each of its constituent NECs to reduce its peak load. The NEC

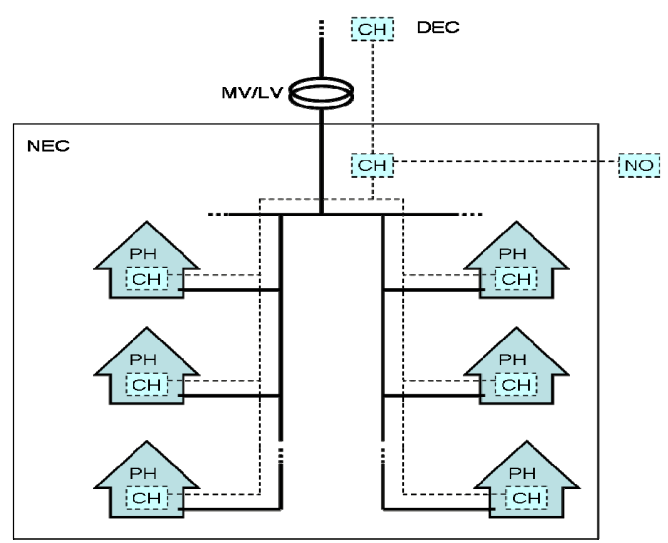

Figure 4. A sketch of the simulation setup $(\mathrm{CH}=$ control holon). 
provides incentives to the PHs to yield peak reduction. For this purpose, we use the dynamic pricing based incentive mechanism that was proposed in [27]. The PHs tends to minimize their cost. Hence, each $\mathrm{PH}$ schedules the (dis)charging of its electric vehicles to minimize its cost in response to the pricing incentive, and then sends its resulting load profile back to the NEC. After receiving the response of all the PHs, the NEC modifies the pricing incentive based on the aggregate load profile and sends it to again to the PHs. This procedure is repeated for a fixed number of iterations. Eventually, the NEC chooses the price vector that yields the lowest peak load, and the corresponding load profiles are adopted. The control agent of the NO employs a low voltage power grid simulator tool called Gaia [28], which is commonly used by network operators in the Netherlands, such as Alliander, to assess the state of the network. For our simulations, we obtained data representing field measurements of the power demand of the appliances as well as the power production of the solar panels and the micro-CHPs from Alliander ${ }^{2}$. Each of these measurements is taken once every 15 minutes over a duration of one day (24 hours). Following this, a time step in our simulation refers to a 15 minutes time interval, and we have 96 time steps during 24 hours. While the patterns of the daily commute distance, the arrival at and departure from home of the EV drivers are derived from the 2009 mobility research of the Netherlands [29], the parameter specifications of the EVs are adopted from [27]. The plots of some of these data are depicted in Figures 5 and 6. Using the proposed control architecture, the following control steps could be involved to achieve the peak minimization in the aggregate load profile of the NEC.

1) The control-holon $(\mathrm{CH})$ of the DEC, the superholon of the NEC, generates and sends a control signal to the $\mathrm{CH}$ of the NEC to reduce its peak load. The $\mathrm{CH}$ of the DEC uses the SteerSubHolon-srv web service to do so.

2) The $\mathrm{CH}$ of the NEC receives the signal from its super-holon using the EnvironmentSA-srv web service, and sets a target state that reduces peak load accordingly, using the TargetState-srv.

3) The $\mathrm{CH}$ of the NEC receives the load profile of its sub-holons (PHs) using the internalDA-srv web service.

4) The CH of the NEC uses the SteerSubHolon-srv web service to generate a price vector based on the aggregate load profile of its sub-holons, and sends it to the CHs of the PHs.

5) The $\mathrm{CH}$ of each $\mathrm{PH}$ receives the price vector from its super-holon using the EnvironmentSA-srv web service, and sets target state that minimizes its cost based on the received price vector, using the TargetState-srv web

\footnotetext{
${ }^{2}$ Alliander is a distribution network operator in The Netherlands owning approximately $40 \%$ of the distribution networks.
}

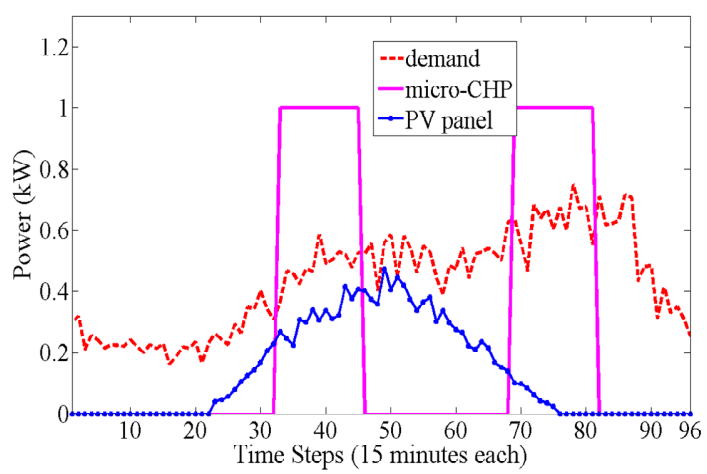

Figure 5. A sample prosumer household demand and production profiles.

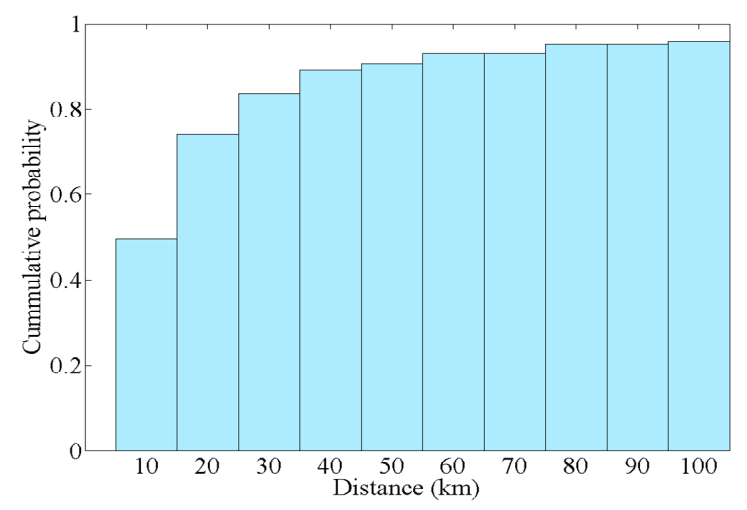

Figure 6. Distribution of the distance traveled per day per car driver.

service.

6) The $\mathrm{CH}$ of each $\mathrm{PH}$ receives the data about the capacity and state of its resources using the InternalDA-srv web service. It then sets its target state, using the TargetStatesrv web service to minimize its cost based on the received price vector, and subsequently schedules, using the Scheduler-srv web service, the (dis)charging of its electric vehicle accordingly. Next, it sends the resulting net load profile of the $\mathrm{PH}$ back to the $\mathrm{CH}$ of the NEC.

7) Steps 3 - 6 represent the negotiation that takes place between the NEC and the PHs to arrive at a situation that yield mutual benefits for the parties. These steps are repeated for a fixed number of iterations. Eventually, the $\mathrm{CH}$ of the NEC selects the price vector that yielded the lowest aggregate peak load, together with the corresponding load profiles.

8) The $\mathrm{CH}$ of the NEC requests the NO to analyze the network state under the resulting load profile.

9) The NO employs the NtkStateAssessment-srv web service to analyze the resulting network state by using Gaia tool. Afterwards, it sends the result of the analysis back to the $\mathrm{CH}$ of the NEC.

10) If the NEC receives a "feasible state" network state report, then the resulting load profile is reported to the $\mathrm{CH}$ of the DEC and the selected price vector is sent 
to the PHs as a final price vector. Subsequently, the $\mathrm{CHs}$ of the PHs use the Device Control-srv web services to control their electric vehicle (dis)charging according to the schedule corresponding to the final price vector.

11) If the received network state is "infeasible state", then the $\mathrm{CH}$ of the NEC will check the network state with the load profiles resulting from the other price vectors until it receives a "feasible state" reply. If none of the load profile yields a healthy network state, then it will generate and send a control signal to the PHs to reduce peak load.

After implementing the web services using the Java Web Services, the above simulation is executed with a NEC consisting of five PHs, and the resulting aggregate load profile is depicted in Figure 7. As can be observed form the figure, coordination of the NEC using our holonic control architecture has yielded a significant reduction (as large as 54\%) in aggregate peak load compared to the uncoordinated scenario where the PHs charge their EVs as soon as they return home from their previous commute. While in our case the Steer Sub Holon-srv service uses the dynamic pricing model proposed in [27] to provide the incentives for the PHs in the NEC, other incentive mechanisms can also be used to achieve a desired aggregate load profile.

\section{Discussions and Conclusions}

The trends of transition in the electric power system are leading to an era of prosumerization whereby myriad of stakeholders autonomously generate and exchange power with each other on the power grid. While the classical power system cannot handle the dynamics since it was designed for centralized generation and unidirectional topdown power flows, the future smart grids are expected to efficiently manage the foreseeable era of prosumerization. To this end, we have proposed to organize the smart grids based on the concepts of holons and holarchy that effectively capture the features of the transitions.

The major features of our holonic smart grids include

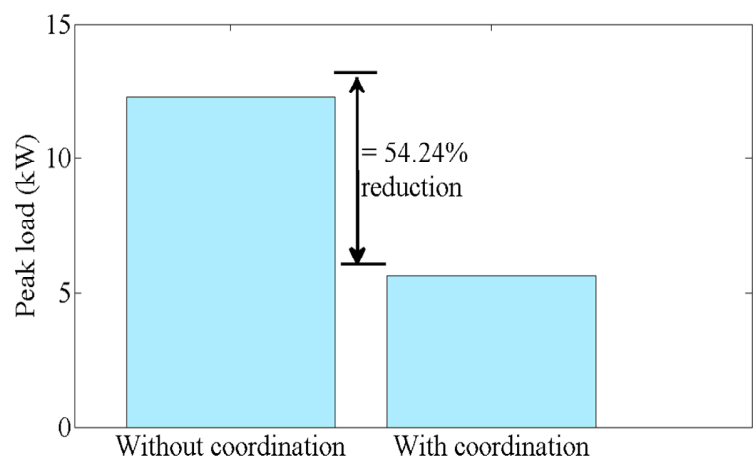

Figure 7. The peak load of a NEC under two scenarios: without coordination and with coordination employing our holonic control architecture. sufficient autonomy of each prosumer to manage its own resources; the recursive structure where a prosumer is composed of smaller prosumers at the lower aggregation layer, and at the same time be part of a larger prosumer at a higher aggregation layer; dynamic reconfiguration to runtime topology change; intelligence and communication capability of prosumers, and maintaining security and privacy of prosumers. The prosumer holons are recursively aggregated into clusters to eventually constitute the smart grid holarchy. Corresponding to the envisaged holonic smart grid, we have proposed a holonic control architecture that conveniently fits the aforementioned features. The control architecture is composed of control-holons corresponding to the prosumer holons that are recursively organized to yield control-holarchy of the smart grid. After specifying relevant control functions of our control-holon, we have defined the corresponding web services based on Service Oriented Architecture (SOA) model. Eventually, the SOA model was implemented using Java web services and tested in a simulation setup yielding interesting results.

In order to implement our proposed smart grid holarchy, there are technical, marketing and regulatory requirements that need to be met. The requirements are in line with the trends in the smart grid developments. One of the requirements is the intelligence and communication capabilities of the prosumers. The current developments in smart meters promise availability of intelligent units that are capable of two way communication [30]. Various developments exist with regards to communication technologies that can be deployed in smart grids, comparisons of the candidate communication technologies are presented in [24]. Another requirement is supporting the bidirectional power flows in the grid. The Future Renewable Electric Energy Delivery and Management (FREEDM) [9] is among the promising developments that address this issue. The capability of dynamic reconfiguration is another requirement. The work presented in [22], which allows a dynamic reconfiguration of a set of microgrids is an interesting development in this regards. With the myriad of prosumers trading electrical power, the challenge lies in how to coordinate the market. The PowerMatcher technology proposed in [10] seems a suitable candidate to adopt for this purpose. Moreover, several solutions have been proposed that can be employed to overcome the security and privacy concerns of the prosumers [24]. Another important requirement is the presence of a suitable regulation that allows the autonomous prosumers to trade power on the grid. Given the trends in the deregulation of electricity markets over the recent past years and the ongoing efforts, although the extents of deregulation vary from one country to another, one can be optimistic to foresee a conducive scenario. 
In general, our proposed smart grid holarchy is a suitable architecture to accommodate the foreseeable era of prosumerization. Moreover, the existing developments and the ongoing efforts in various aspects of the smart grids are in line with the requirements for its implementation.

\section{REFERENCES}

[1] International Energy Agency, “Technology Roadmaps Smart Grids,” 2011.

http://www.iea.org/publications/freepublications/publicati on/smartgrids_roadmap.pdf

[2] National Institute of Standards and Technology, "NIST Framework and Roadmap for Smart Grid Interoperability Standards, Release 1.0,” 2010.

http://www.nist.gov/public_affairs/releases/upload/smartg rid_interoperability_final.pdf

[3] European Smart Grids Technology Platform, "Vision and Strategy for Europe's Electricity Networks of the Future," 2006.

ftp://ftp.cordis.europa.eu/pub/fp7/energy/docs/smartgrids _en.pdf

[4] K. Dielmann and A. van der Velden, "Virtual Power Plants (VPP)—A New Perspective for Energy Generation?" Proceedings of the 9th International Scientific and Practical Conference on Modern Techniques and Technologies, 2003, pp. 18-20.

[5] N. Hatziargyriou, N. Jenkins, G. Strbac, J. A. P. Lopes, J. Ruela and A. Engler, "MICROGRIDS-Large Scale Integration of Micro-Generation to Low Voltage Grids," CIGRE C6-309, 2006.

[6] F. Provoost, J. Myrzik and W. Kling, "Setting Up Autonomous Controlled Networks," $39^{\text {th }}$ International Universities Power Engineering Conference (UPEC), Vol. 3, 2004, pp. 1190-1194.

[7] F. Overbeeke and V. Roberts, "Active Networks as Facilitators for Embedded Generation," Cogeneration and On-Site Power production, Vol. 3, No. 2, 2002, pp. 3742.

[8] L. Tsoukalas and R. Gao, "From Smart Grids to an Energy Internet: Assumptions, Architectures and Requirements,” 3rd International Conference on Electric Utility Deregulation and Restructuring and Power Technologies, Nanjing, May 2008, pp. 94-98. doi:10.1109/DRPT.2008.4523385

[9] A. Huang, M. Crow, G. Heydt, J. Zheng and S. Dale, "The Future Renewable Electric Energy Delivery and Management (FREEDM) System: The Energy Internet," Proceedings of the IEEE, Vol. 91, 2011, pp. 133-148.

[10] J. Kok, C. Warmer and I. Kamphuis, "Power Matcher: Multiagent Control in the Electricity Infrastructure," Proceedings of the 4th International Joint Conference on Autonomous Agents and Multiagent Systems, Utrecht, 2529 July 2005, pp. 75-82.

[11] A. Vaccaro, M. Popov, D. Villacci and V. Terzija, “An Integrated Framework for Smart Microgrids Modeling, Monitoring, Control, Communication, and Verification," Proceedings of the IEEE, Vol. 99, 2011, pp. 119-132.
[12] A. Koestler, “The Ghost in the Machine,” Penguin Group, New York, 1990.

[13] G. Zhang and W. Li, "Flexible Holonic Organization Modeling and Cultural Evolution," 4th IEEE International Conference on Wireless Communications, Networking and Mobile Computing, Dalian, 12-14 October 2008, pp. 1-5.

[14] H. Van Brussel, J. Wyns, P. Valckenaers, L. Bongaerts and P. Peeters, "Reference Architecture for Holonic Manufacturing Systems: PROSA,” Computers in Industry, Vol. 37, No. 3, 1998, pp. 255-274. doi:10.1016/S0166-3615(98)00102-X

[15] M. Moghadam and N. Mozayani, “A Street Lighting Control System Based on Holonic Structures and Traffic System,” IEEE 3rd Inernational Conference on Computer Research and Development (ICCRD), Shanghai, 11-13 March 2011, pp. 92-96.

[16] Y. Ye, V. Hilaire, A. Koukam and C. Wandong, “A Holonic Model in Wireless Sensor Networks,” IEEE International Conference on Intelligent Information Hiding and Multimedia Signal Processing, Harbin, 15-17 August 2008, pp. 491-495. doi:10.1109/IIH-MSP.2008.37

[17] N. Baken, "Holons and Holarchies,” TEDx Amsterdam 2009.

http://www.tedxamsterdam.com/2009/video-nico-bakeno n-holons-and-holarchies/

[18] D. O’Neill, M. Levorato, A. Goldsmith and U. Mitra, "Residential Demand Response Using Reinforcement Learning," 1st IEEE International Conference on Smart Grid Communications, Gaithersburg, October 2010, pp. 409-414.

[19] C. Ibars, M. Navarro and L. Giupponi, "Distributed Demand Management in Smart Grid with a Congestion Game,” 1st IEEE International Conference on Smart Grid Communications, Gaithersburg, 4-6 October 2010, pp. 495-500.

[20] E. Gerding, V. Robu, S. Stein, D. Parkes, A. Rogers and N. Jennings, “Online Mechanism Design for Electric Vehicle Charging,” 10th International Joint Conference on Autonomous Agents and Multi-Agent Systems, Taipei, 2-8 May 2011, pp. 811-818.

[21] H. Simon, “Architecture of Complex Systems,” Proceedings of the American Philosophical Society, Vol. 106, No. 6, 1962, pp. 467-482.

[22] M. Erol-kantarci, B. Kantarci and H. Mouftah, "Reliable Overlay Topology Design for the Smart Microgrid Network,” IEEE Transactions on Networks, Vol. 25, No. 5, 2011, pp. 38-43. doi:10.1109/MNET.2011.6033034

[23] Alstom Grid, "Network Protection and Automation Guide,” 2011.

[24] X. Fang, S. Misra, G. Xue and D. Yang, "Smart GridThe New and Improved Power Grid: A Survey," IEEE Communication Surveys and Tutorials, Vol. 14, No. 4, 2011, pp. 944-980. doi:10.1109/SURV.2011.101911.00087

[25] M. A. Lisovich and S. B. Wicker, "Privacy Concerns in Upcoming Residential and Commercial Demand-Response Systems," Proceedings of the Clemson University Power 
Systems Conference, 2008.

[26] D. M. Han and J. H. Lim, "Design and Implementation of Smart Home Energy Management Systems Based on Zigbee,” IEEE Transactions on Consumer Electronics, Vol. 56, No. 3, 2010, pp. 1417-1425. doi:10.1109/TCE.2010.5606278

[27] E. Negeri and N. Baken, "Smart Integration of Electric Vehicles in an Energy Community," Proceedings of the 1st International Conference on Smart Grids and Green IT Systems, Porto, 19-20 April 2012, pp. 25-32.
[28] The Gaia Tool. http://www.phasetophase.nl/en_products/vision_lv_netwo rk_design.html

[29] MON, “Mobility Research of the Netherlands," 2009. http://rijkswaterstaat.nl/

[30] L. Wang, V. Devabhaktuni and N. Gudi, "Smart Meters for Power Grid-Challenges, Issues, Advantages and Status,” IEEE/PES Power Systems Conference and Exposition (PSCE), Phoenix, 20-23 March 2011. 